\title{
The Dark Energy Survey
}

\author{
Ignacio Sevilla*t \\ Centro de Investigaciones Energeticas MedioAmbientales y Tecnologicas, CIEMAT, Spain \\ E-mail: ignacio.sevilla@ciemat.es
}

\begin{abstract}
The discovery that the universe is accelerating, not slowing down from the mass it contains, is the surprise that sets the initial research program of 21st Century cosmology. The Dark Energy Survey is a next generation sky survey aimed directly at understanding this mystery. We will build an extremely red sensitive 500 Megapixel camera, with a 1 meter diameter, 2.2 degree field of view prime focus corrector, and a data acquisition system fast enough to take images in 17 seconds. The cage containing the system mounts at the prime focus of the Blanco 4-meter telescope at Cerro Tololo Interamerican Observatory (CTIO) in Chile. Over 5 years we will use $30 \%$ of the available time on the telescope to pursue a high precision multi-bandpass wide area survey, designed to produce photometric redshifts from $0.2<\mathrm{z}<1.3$. The survey g,r,i,z,Y data will cover 5000 sq-degrees, with 4000 sq-degrees overlapping the Sunyaev-Zeldovich CMB survey being conducted by the South Pole Telescope. Our 4 science goals aim at extracting cosmological information on the dark energy from 1) cluster counting and spatial distribution of clusters at $0.1<\mathrm{z}<1.3,2$ ) the shifting of the galaxy spatial angular power spectra with redshift, 3 ) weak gravitational lensing measurements on several redshift shells to $z \sim 1$, and 4) 2000 type Ia supernovae at $0.3<\mathrm{z}<0.8$. The signature of dark energy being a cosmological constant is that the dark energy density remains constant while the universe expands; technically that $\mathrm{w}=-1$ and that $\mathrm{dw} / \mathrm{dt}=0$. We aim at a $5 \%-15 \%$ precision measurement in $\mathrm{w}$ from each of our experiments, and a $30 \%$ measurement in w'. Combined, they provide both stronger constraints and a check on systematic errors.
\end{abstract}

European Physical Society Europhysics Conference on High Energy Physics, EPS-HEP 2009, July 16 - 222009

Krakow, Poland

\footnotetext{
* Speaker.

$\dagger$ on behalf of the DES Collaboration
} 


\section{Introduction}

The Dark Energy Survey (DES) is a project designed to perform a deep optical-near infrared survey of 5000 square degrees of the South Galactic Cap to $\sim 24$ th magnitude using the grizY filters. The goal is to measure the properties of dark energy through the determination of its equation of state using four independent methods: galaxy clusters, weak gravitational lensing tomography, galaxy angular clustering and type Ia supernova distances [1][2]. The obvious advantage of this approach is to have the same instrument making the four measurements providing important information on the systematic errors.

\section{The Project}

DES arose from an announcement of opportunity issued by the National Optical Astronomy Observatory in 2003 to build a new instrument for the Blanco Telescope in exchange of 30\% of the observation time during a 5-year period. The project includes the building of the camera (DECam [3]), a data management system and improvements at the telescope site. The plan is to start scientific operations in Fall 2011.

\section{Dark Energy}

The evidence for dark energy is two-fold (see also [6]):

- The accelerated expansion of the Universe, measured with supernovae type Ia [4].

- The Universe is flat, but matter content is $27 \%$ (from large scale structure measurements).

This leads to two alternatives:

- An additional unknown stress-energy component is present with negative pressure.

- General relativity breaks down at large distances or the cosmological principle fails.

A phenomenological way to parametrize dark energy properties is to measure its equation of state [5]: $w=p / \rho$. The main features to be tested are: is $w$ compatible with 1? Is $d w / d z$ compatible with zero?

\section{The Probes}

\subsection{Galaxy Clusters}

The cluster number count per unit volume is [7]:

$$
\frac{d^{2} N}{d z d \Omega}=\frac{c}{H(z ; \theta, w)} D_{A}^{2}(1+z)^{2} \int_{0}^{\infty} f(O, z) d O \int_{0}^{\infty} p(O \mid M, z) \frac{d n}{d M}(z ; \theta) d M
$$

where $H$ is the Hubble constant, $D_{A}$ is the angular diameter distance of the volume bin, $f(O, z)$ is the observable function, $p(O \mid M, z)$ is the observable-mass relationship and $d n / d M(z, \theta)$ is the cluster mass function. This a theoretically very sensitive probe of structure formation. By obtaining 
the statistics of cluster at each redshift bin and cluster mass predictions, it is possible to obtain the best cosmology which fits the previous equation. The idea in DES is to measure up to $\sim 20000$ clusters using the partnership with the South Polar Telescope for cluster identification. Though promising, this technique has to cope with difficult systematic error sources from the observablemass relationship and the sample purity and completeness.

\subsection{Weak Lensing Tomography}

This technique is another structure probe [8], which relies on the statistical measurement of distortions of background objects created by intervening matter (shear-shear measurements). Another handle would be to measure cross-correlations between the identified foreground galaxies and nearby shear (galaxy-shear measurements). Additionally, the shear angular power spectrum can provide yet another cosmologically linked measurement. DES will make the shear measurements for $\sim 3 \cdot 10^{8}$ galaxies, with PSF shapes under 0.9 ". This method is very well-founded by the underlying physics, though affected very much by uncontrolled PSF, the determination of photometric redshifts and shear calibration.

\subsection{Galaxy Angular Clustering}

The cosmological microwave background provides the scale of the acoustic peak. The search for this peak in the angular two-point correlation function of certain types of galaxies in different redshift shells is the basis of the method. These are the so-called baryonic acoustic oscillations (BAO) [9]. They give an estimation of the expansion history, and ultimately, of the cosmological parameters including $w$. DES will provide the power spectrum of $\sim 3 \cdot 10^{8}$ galaxies up to $\mathrm{z} \sim 1.5$. The method is very straightforward to apply, though the constraints provided by it are the weakest. The most notable systematic effects are the photometric redshift errors, the projection effects of "collapsing" thick volumes into redshift shells and the effect of non-linear evolution.

\subsection{Supernova Luminosity Distance}

By obtaining the light curves (photometry with time) of many hundreds of supernovae, we can relate them to their intrinsic luminosity which, combined with their apparent magnitude and redshift, can be used to minimize the following $\chi^{2}$ function, which depends on the luminosity distance $d_{L}$ :

$$
\chi^{2}=\sum_{\text {objects }} \frac{\left.(\mu-5 \log )\left(d_{L}(z ; \theta, w)\right) / 10 p c\right)^{2}}{\sigma^{2}}
$$

where $\mu$ is the distance module. DES will measure a few thousands of supernovae photometrically, up to redshift $z=1$. This is in part thanks to its improved $z$-band response. $10 \%$ of the survey time will be devoted to supernovae search revisiting an area of 40 square degrees. Photometric redshift errors will be addressed with very precise on-site measurements of photometry, and spectroscopic follow-ups of a significant fraction of the events.

\section{Expected Performance}

Figure 1 shows a plot of the expected constraints in the parameters $w_{a}$ and $w_{0}$, considering the Linder parametrization [10] and assuming $\Lambda \mathrm{CDM}$ : 


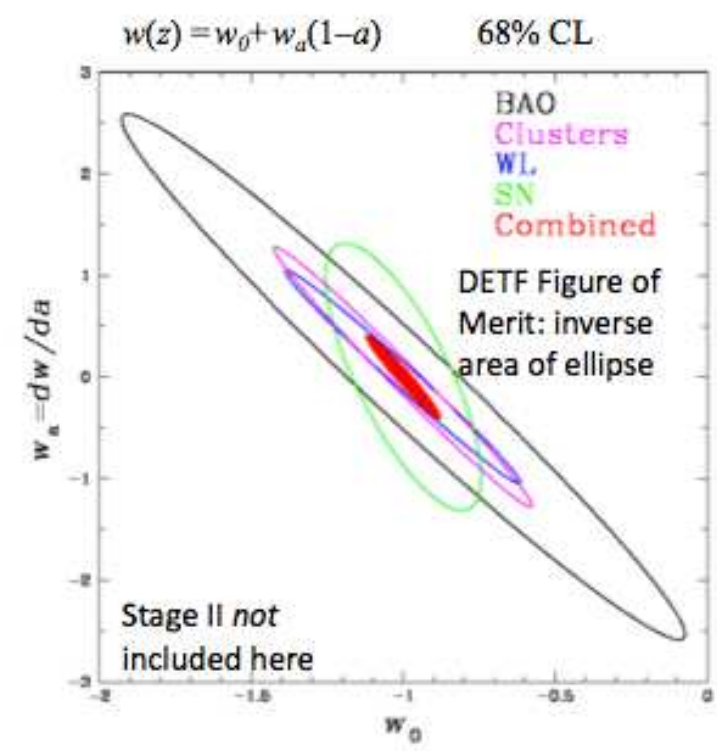

Figure 1: Expected performances on the equation of state parameters $w_{a}$ and $w_{0}$ for DES.

Only statistical and photo-z systematic errors have been considered. For BAO, a Planck CMB prior has been used.

\section{References}

[1] A. Albrecht et al., Report of the Dark Energy Task Force, ast ro-ph/0609591

[2] The Dark Energy Survey Collaboration, The Dark Energy Survey, astro-ph / 0510346

[3] I. Sevilla, The Dark Energy Survey, these proceedings.

[4] S. Perlmutter et al., Measurements of Omega and Lambda from 42 High-Redshift Supernovae ApJ 517 (1999) 565; A. Riess et al., Observational Evidence from Supernovae for an Accelerating Universe and a Cosmological Constant Astron. J 116 (1998) 1009

[5] M. Turner, M. White, CDM Models with a Smooth Component, Phys.Rev.D 56 (1997) 4439

[6] P. Astier, Cosmology and Dark Energy, these proceedings.

[7] J. Frieman, M. Turner, D. Huterer, Dark Energy and the Accelerating Universe, Ann.Rev.Astron.Astrophys. 46 (2008) 385

[8] W. Hu, Dark Energy and Matter Evolution from Lensing Tomography Phys.Rev.D, 66 (2002) 083515 (astro-ph / 0208093 ); D. Huterer, Weak Lensing and Dark Energy, Phys.Rev.D, 65 (2002) 06300 (astro-ph/0106399)

[9] H-J. Seo, D. Eisenstein, Probing Dark Energy with Baryonic Acoustic Oscillations from Future Large Galaxy Redshift Surveys, emphApJ 598 (2003) 720 (astro-ph/0307460)

[10] E. Linder, Exploring the Expansion History of the Universe, Phys. Rev. Lett.90 (2003) 091301, (astro-ph/0208512) 